\title{
The FOSS 2010 Community Report
}

\author{
Walt Scacchi ${ }^{1}$, Kevin Crowston ${ }^{2}$, Greg Madey ${ }^{3}$, and Megan Squire ${ }^{4}$ \\ ${ }^{1}$ University of California, Irvine, Institute for Software Research, \\ Irvine, CA, USA \\ wscacchi@ics.uci.edu \\ http: / /www.ics.uci.edu/ wscacchi \\ ${ }^{2}$ Syracuse University, School of Information studies, Syracuse, NY, USA \\ crowstonasyr.edu, \\ http: / / crowston.syr.edu/ \\ ${ }^{3}$ University of Notre Dame, South Bend, IN, USA \\ gmadey@nd.edu \\ http: / /www. nd.edu/ gmadey / \\ ${ }^{4}$ Elon University, Department of Computing Sciences, \\ Elon, NC, USA \\ msquire@elon.edu \\ http: //facstaff.elon.edu/msquire
}

\begin{abstract}
The purpose of this panel is to disseminate the findings from the related FOSS workshop, a CCC-sponsored exploratory workshop held at University of California, Irvine in February 2010. At the OSS conference we will give first a report of what was learned at the FOSS workshop, and then we will glean important feedback from community members who were unable to be at the FOSS workshop. The four conveners of the FOSS workshop will be the panelists at the OSS conference.
\end{abstract}

\section{The Purpose of the Workshop}

The purpose of the FOSS workshop at UC-Irvine was to generate ideas and perspectives from within the free and open source research community about the future of research in the field. To start the workshop, we solicited position papers from our fifty North American attendees about the following subject areas:

- How does FOSS as a diverse socio-technical movement accomplish global software development, without a traditional central authority or source of funding/resources?

- How do distributed groups make decisions? What sort of conflicts are common, and how are conflicts settled?

- What are the differences and similarities between FOSS projects and proprietary (non-FOSS) projects? Is there a taxonomy of characteristics of these two types of projects? Are there hybrid projects, and how are these described?

- How do we measure "success" of a FOSS project? What are the various attributes of a project that might help us measure success? Do we have all the data we need, or are there additional measures that we need to collect? 
- What are the different ways that software developers are incentivized within the various types of FOSS projects? How does this incentive structure compare to proprietary projects? What do the developers themselves report are the best and worst incentives?

- How can the benefits of FOSS be translated into a language technology decision-makers can understand? Are there "best practices" for FOSS technology adoption or for rollovers from proprietary to FOSS models within businesses or governments?

- What are the various techniques and technologies that help self-organized groups to work effectively? How can these self-organizing techniques and technologies be applied to other domains?

- What are the different roles in a FOSS project (e.g., core developer, active user)? What levels of contribution is needed from members in various roles are needed to sustain a project (e.g., how important are active users)?

- How long can such a movement be sustained?

- Are there conditions or events that constitute an inflection point that will mark the decline of FOSS as a socio-technical movement?

\section{Findings from the Workshop}

Based on discussions, debate, and reflection at the FOSS workshop, we were able to synthesize and change the list of questions and focus areas. At the end of the FOSS workshop, our focus areas for the future of FOSS research included:

- Collaboration - how can studying FOSS help us understand how humans collaborate?

- Software Engineering Practice - how can FOSS help us understand the current and future state-of-the-art in software development?

- Transfer, Ecosystem, and Society - what can we learn about other domains from studying FOSS, and from what other domains is FOSS being influenced?

- Learning and Education - can FOSS serve as an educational tool and what are the implications of using FOSS in the classroom?

- Evolution - how do FOSS outcomes, activities, technologies, infrastructures, etc. develop and change over time? Do these changes follow specific patterns or principles, and what evolutionary trajectories are typical and or similar within FOSS when compared to other forms of developing and evolving software?

- Motivational Transformations - how does studying FOSS help us understand the global IT infrastructure and the process of innovation?

- Research Infrastructures - what are the best ways to support the data and analysis needs of the research community?

These interest areas represent what the community believes will be the best way to focus and extend the FOSS research agenda in the coming years. 